\title{
POSITRON ANNIHILATION WITH CORE ELECTRONS IN 3d-TRANSITION METALS
}

\author{
A. Baranowski and E. Dębowska \\ Institute of Experimental Physics, University of Wrocław \\ Maxa Borna 9, 50-204 Wrocław, Poland
}

\begin{abstract}
Recently- we have been able to resolve the rare gas core contribution in long-slit angular correlation of annihilation radiation curves for some $d$-metals when applying a new model to fit the experimental data. In the present paper the model is applied to angular correlation of annihilation radiation spectra for the whole series of the $3 d$-transition metals from Sc to $\mathrm{Cu}$. The model corresponds to recent theoretical predictions for the metals from the second half of the series and allows one to find the contribution of the core electrons to the whole annihilation process for these metals. Probably a different description of the angular correlation of annihilation radiation data for the metals from the beginning of the $3 d$-series should be searched.
\end{abstract}

PACS numbers: $78.70 . \mathrm{Bj}, 71.60 .+\mathrm{z}, 71.90 .+\mathrm{q}$

\section{Introduction}

Experimental one-dimensional angular correlation of annihilation radiation (1D-ACAR) data coming from the $2 \gamma$ quanta annihilation of the positron-electron pairs in any metallic sample deviate from a parabolic shape expected for the ideal Fermi gas. For simple polycrystalline metals the data are usually fitted by the sum of an inverted parabola and a gaussian. In this representation the parabolic part is considered to be due to valence electrons, whereas the gaussian part corresponds to the annihilation with the rare gas core electrons. As early as in 1971 West [1] suggested that only about $80 \%$ of the gaussian corresponds to the proper core annihilation and the remainder comes from the contribution of the high momentum components (HMC) of valence electrons. The suggestion has been next confirmed by Sob's calculations $[2,3]$ showing that the HMC contribution to the gaussian part of $A C A R$ for simple metals is not negligible anyway and amounts from $23 \%$ in $\mathrm{Na}$ to $34 \%$ in $\mathrm{Cs}$ and is even larger for polyvalent simple metals $(41 \%$ in $\mathrm{Mg}$ ). Such a conclusion results from the values of the $\lambda_{\text {HMC }} /\left(\lambda_{\mathrm{c}}+\lambda_{\text {HMC }}\right)$ ratio, where $\lambda_{\mathrm{HMC}}$ and $\lambda_{c}$ are the HMC and core annihilation rates calculated in the above papers. 
In Sob's calculations it was taken into account that the experimental annihilation rate is higher than that predicted by the independent particle model (IPM) due to enhanced electron density at the positron position resulting from the electron-positron interaction. The enhancement effect has been intensively studied by a number of authors but unfortunately the unified theory of the enhanced annihilation with different groups of electrons in real metals has not been created up to now. According to the formula given by Sob [3] the ratios of the average enhancement factor (EF) for valence and core electrons in simple metals are larger or equal to 1.8 . Hence in these metals the core and valence electrons are clearly distinguishable with respect to the positron behaviour. Not very much is known about the momentum dependence of EF for core electrons. The EF independence of momentum was found in Ref. [4] while the local density approach (LDA) [5] applied to describe positron annihilation with core electrons in alkalis led to momentum dependent EF with large difference in the low-momentum $(p \leq 15-20 \mathrm{mrad})$ and high-momentum $(p \geq 20 \mathrm{mrad})$ regions. The average values of $\mathrm{EF}$ for low-momentum and high-momentum regions are in very good agreement with those obtained in Ref. [2]. As the HMC of valence electrons contribute a lot essentially in the same momentum region as core electrons the HMC enhancement factor should also be known. The enhancement effect in the (110) HMC zones in $\mathrm{Li}$ and $\mathrm{K}$ appeared to be similar in magnitude to that in the central zone [6]. In their empirical approaches Šb [7, 8] and independently Mijnarends and Singru [9] proposed the energy-dependent EF, which was quite successful when applied in the local density approximation to describe electron-positron interaction (for a recent review see [10]).

Not everything is clear as far as the EF with different electron groups in simple metals is concerned but the situation is worse when $d$-metals are taken into account. At present two methods, local density approach and Bloch-modified ladder approach are used in treating the $e-p$ interaction with $d$-electrons, but a true many-body theory of positron annihilation with $d$-electrons is needed to find a better agreement with experiment in both lifetime and angular correlation results.

The annihilation rate with rare gas core ( $\mathrm{rgc}$ ) electrons in $d$-metals has been thought to constitute only few per cent of the total rate [11]. The author of Ref. [3] calculated, however, that in $3 d$-and $4 d$-metals the rgc part of the total annihilation rate amounts to $9 \%$ in $\mathrm{Zn}$ and even to $37 \%$ in Sc. It is worthy to mention also the paper [12], where the momentum densities of $e-p$ pairs, positron annihilation rates, and $e-p$ enhancement factors for rgc electrons in 27 simple and $d$-metals were determined using various parameters in the calculation of electron and positron wave functions. The LDA method used in Ref. [12] led to a fairly good agreement of the calculated total annihilation rates with the experimental ones. The rgc contribution to the total annihilation rate, according to the results of Ref. [12], changes from about $25 \%$ for $\mathrm{Sc}-\mathrm{Cr}$ to over a dozen percent for $\mathrm{Fe}-\mathrm{Cu}$.

The contribution of the rare gas core annihilation to the 1D-ACAR for noble and $d$-transition metals has usually been neglected and the simple parabola and gaussian $(P+G)$ model has been used to fit the data although the values of the normalized $\chi^{2}$ have never been close to 1 . The parabolic part of the curve for these metals was thought to originate from valence electrons, while the gaussian part 
was ascribed mainly to $d$-electron annihilation. The contribution of the valence electron HMC to the gaussian has never been explicitly calculated in $d$-metals nor has been extracted from the experimental ACAR data.

In Ref. [13] a profound analysis of long-range ACAR data for some $d$-transition and noble metals was presented. We aimed to find three different functions describing the annihilation with three different electron groups, the nearly free (NF), $d$-like, and rare gas core electrons. The annihilations with NF and rgc electrons were assumed to be represented by a parabola $P$ and a wide gaussian $G_{\mathrm{W}}$, respectively, as in the case of simple metals. The $d$-electron part of the ACAR data was described by a so-called Ferrell function $F$ being the product of a gaussian and a biquadratic polynomial. The $P+F+G_{\mathrm{W}}$ fit, unsuccessful when applied to short-range data of $(-23,23)$ mrad, turned out to be a very good one if applied to the long-range data of $(-35,35) \mathrm{mrad}$. As far as we know, this was the first experimental evidence of rare gas core annihilation contribution to the 1D-ACAR in $d$-metals. The new model was next successfully applied to ACAR data for Pd-Ag. and $\mathrm{Pd}-\mathrm{Au}$ [14] as well as $\mathrm{Pd}-\mathrm{Ag}-\mathrm{H}$ and $\mathrm{Pd}-\mathrm{Au}-\mathrm{H}$ [15] alloys allowing the investigation of the electronic structure of those systems. In the present paper the model is applied to ACAR data for the whole series of the $3 d$-transition metals with the aim of establishing the contribution of rare gas core electrons (the value of the parameter $G_{\mathrm{W}}$ ) to the total angular correlation curve.

\section{Measurements and results}

The samples of $3 d$-transition metals for the ACAR measurements were prepared by a standard procedure. Pure metals (the purity is given in Table I) were melted under argon atmosphere in an arc furnace except for Sc and Co that were bought in the shape of metal plate. The melts were cut into two hemispheres and the surfaces were polished mechanically. All the samples were subjected to annealing at an appropriate temperature (see Table $\mathrm{I}$ ); for $\mathrm{V}$ and $\mathrm{Cr}$, with higher melting temperature, the graphite furnace was used. The effect of annealing was controlled by positron lifetime measurements using a spectrometer with 330 ps time resolution. The data were analysed by Positronfit program and the resulting lifetimes are collected in Table I. We also show the positron lifetime values obtained by other authors. From the comparison it follows that our value for Mn is too high; it does not fit into the values for the other metals, either. But generally our positron lifetime values allowed us to state that the samples were. well prepared to angular correlation measurements.

The ACAR data were obtained using the long-slit geometry apparatus with the angular resolution of $1 \mathrm{mrad}$. The measurements were carried out in the range of $(-37,37) \mathrm{mrad}$ with $0.5 \mathrm{mrad}$ step. The number of counts at maximum was about (5-8) $\times 10^{4}$. The $P+F+G_{\mathrm{W}}$ model, as we mentioned in Sec. 1, proved to be a very good one for some $d$-transition and noble metals, so we have tried to check its applicability to whole $3 d$-metal series. Some parameters of the fit are given in Table II. From the point of view of the $\chi^{2}$ value, the model works well for all the metals except of Sc. The examples of the $P+F+G_{\mathrm{w}}$ fit to ACAR data and the normalized differences between the experimental data and the model for 
TABLE I

Positron lifetime for $3 d$-transition metals.

\begin{tabular}{c|c|c|c|c}
\hline \hline Metal & Purity & $T_{\mathrm{a}}$ & \multicolumn{2}{|c}{$\tau[\mathrm{ps}]$} \\
\cline { 4 - 5 } & {$[\mathrm{N}]$} & {$[\mathrm{K}]$} & $\mathrm{a}$ & $\mathrm{b}$ \\
\hline $\mathrm{Sc}$ & 3 & 1370 & $190 \pm 2$ & 230 \\
$\mathrm{Ti}$ & 4 & 1270 & $151 \pm 2$ & 147 \\
$\mathrm{~V}$ & 5 & 1870 & $123 \pm 1$ & 130 \\
$\mathrm{Cr}$ & 4 & 1870 & $112 \pm 1$ & 120 \\
$\mathrm{Mn}$ & 3 & 1170 & $155 \pm 2$ & 129 \\
$\mathrm{Fe}$ & 5 & 1370 & $108 \pm 2$ & 106 \\
$\mathrm{Co}$ & 5 & 1370 & $113 \pm 1$ & 118 \\
$\mathrm{Ni}$ & 5 & 1370 & $108 \pm 2$ & 110 \\
$\mathrm{Cu}$ & 4 & 1070 & $118 \pm 2$ & 110 \\
\hline
\end{tabular}

$T_{\mathrm{a}}$ - annealing temperature, $\tau$ - positron bulk lifetime; column a - this work, column b - Ref. [16] for Sc and $\mathrm{Co}$, Ref. [17] for Mn, and Ref. [18] for other metals.

TABLE II

Experimental positron annihilation characteristics for $3 d$-transition metals.

\begin{tabular}{l|c|r|r|r|c|c}
\hline \hline Metal & $\chi^{2}$ & \multicolumn{1}{c|}{$\begin{array}{c}P F \\
{[\%]}\end{array}$} & \multicolumn{1}{|c|}{$\begin{array}{c}G_{\mathrm{W}} F \\
{[\%]}\end{array}$} & $\begin{array}{c}\lambda_{c} / \lambda_{\exp } \\
{[\%]}\end{array}$ & $\begin{array}{c}x_{\mathrm{p}} \\
{[\mathrm{mrad}]}\end{array}$ & $\begin{array}{c}N_{\mathrm{NF}} \\
{[\mathrm{el} / \mathrm{at}]}\end{array}$ \\
\hline $\mathrm{Sc}$ & 2.20 & $7.1 \pm 0.2$ & $48.3 \pm 1.1$ & 23.1 & $2.92 \pm 0.03$ & $0.36 \pm 0.01$ \\
$\mathrm{Ti}$ & 1.30 & $3.6 \pm 0.3$ & $32.5 \pm 0.3$ & 25.0 & $5.64 \pm 0.11$ & $1.85 \pm 0.11$ \\
$\mathrm{~V}$ & 1.18 & $3.3 \pm 0.2$ & $24.9 \pm 0.6$ & 24.4 & $5.45 \pm 0.17$ & $1.31 \pm 0.13$ \\
$\mathrm{Cr}$ & 1.28 & $4.6 \pm 0.2$ & $11.8 \pm 0.4$ & 23.4 & $6.18 \pm 0.10$ & $1.65 \pm 0.08$ \\
$\mathrm{Mn}$ & 0.86 & $3.5 \pm 0.2$ & $8.6 \pm 0.4$ & 26.4 & $5.95 \pm 0.13$ & $1.50 \pm 0.10$ \\
$\mathrm{Fe}$ & 1.12 & $8.6 \pm 0.3$ & $14.2 \pm 0.7$ & 16.8 & $5.91 \pm 0.06$ & $1.42 \pm 0.06$ \\
$\mathrm{Co}$ & 1.01 & $7.8 \pm 0.2$ & $16.9 \pm 0.6$ & 16.3 & $6.00 \pm 0.06$ & $1.40 \pm 0.05$ \\
$\mathrm{Ni}$ & 1.10 & $8.6 \pm 0.2$ & $13.8 \pm 0.4$ & 14.0 & $5.96 \pm 0.05$ & $1.35 \pm 0.04$ \\
$\mathrm{Cu}$ & 1.10 & $14.1 \pm 0.3$ & $11.7 \pm 0.6$ & 11.7 & $5.57 \pm 0.03$ & $1.19 \pm 0.04$ \\
\hline
\end{tabular}

$\chi^{2}-$ goodness of the $P+F+G_{\mathrm{W}}$ fit, $P F-$ parabola fraction, $G_{\mathrm{w}} F$ - wide gaussian fra $\lambda_{\mathrm{c}} / \lambda_{\exp }$ - the ratio of the core annihilation rate [12] to the experimental rate, $x_{\mathrm{p}}$ - parabola cut-off, $N_{\mathrm{NF}}-$ number of nearly free electrons.

Sc and Co are presented in Fig. 1 and Fig. 2, respectively. From the tendencies in the fitting (Fig. 1b) incorrectness of the model for Sc is clearly visible. It should be stressed however that the results for $\mathrm{Ti}$ are sensitive to the starting fit parameters; some sensitivity is also observed for $\mathrm{V}$.

As we have pointed out earlier, the wide Gaussian in $P+F+G_{\mathrm{w}}$ model is thought to represent the contribution from the positron annihilation with the rare 

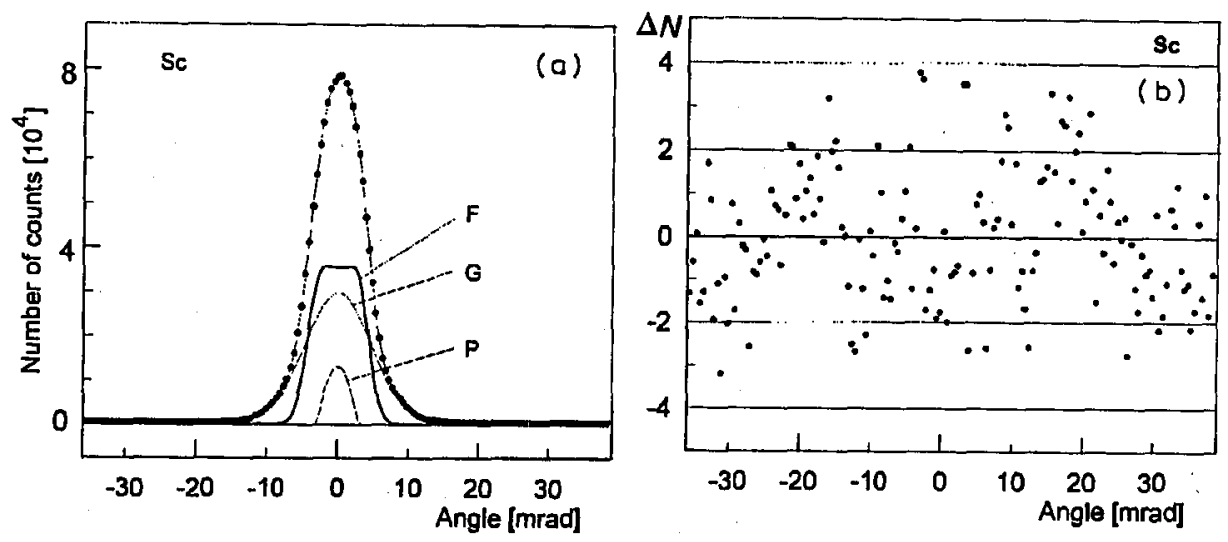

Fig. 1. The $P+F+G_{w}$ model to ACAR data for Sc; (a) fitting of the model, (b) normalized differences $\Delta N$ between experimental and model data.
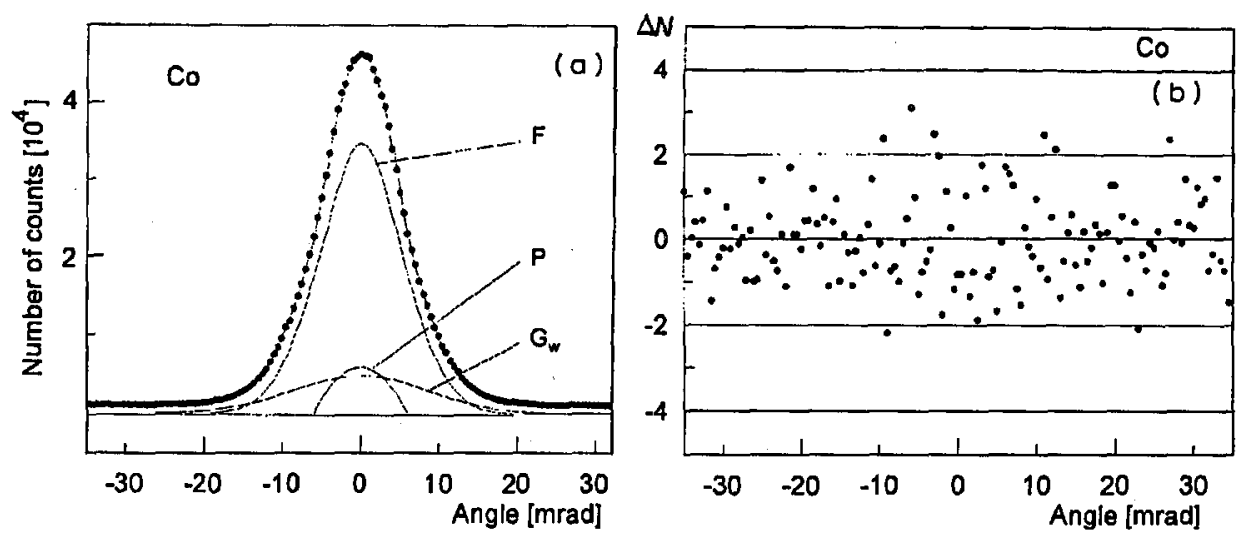

Fig. 2. The $P+F+G_{\mathrm{w}}$ model to ACAR data for Co; (a) fitting of the model, (b) normalized differences $\Delta N$ between experimentăl and model data.

gas core electrons. Its fraction, the parameter $G_{\mathrm{W}} F$ in Table II, can be compared with a ratio of the calculated core annihilation rate $\lambda_{c}$ to the experimental rate $\lambda_{\text {exp }}$. In Table II such a comparison is presented with $\lambda_{c}$ values taken from Ref. [12] and $\lambda_{\exp }$ from our lifetime measurements. We employed here the value of $\lambda_{\mathrm{c}}^{0}$ resulting from the calculations with a solid state electron configuration and unperturbed positron wave function; the other calculated $\lambda_{\mathrm{c}}$ values do not differ too much [12]. An excellent agreement between $G_{\mathrm{W}} F$ and $\lambda_{c} / \lambda_{\exp }$ for $\mathrm{V}, \mathrm{Co}, \mathrm{Ni}$ and $\mathrm{Cu}$, and quite a good one for $F e$ was found. For the other metals some differences appear; whereas for $\mathrm{Ti}$ the difference between theory and experiment is not big, for Sc the $G_{\mathrm{W}} F$ is twice as large as $\lambda_{\mathrm{c}} / \lambda_{\mathrm{exp}}$, and for $\mathrm{Cr}$ and $\mathrm{Mn}$ amounts to about one half and one third of $\lambda_{c} / \lambda_{\text {exp }}$, respectively. Thus the model works much better for the metals from the second half of the series than from the first one. It seems that another function than wide gaussian should be found to describe core contribution 
to ACAR for the metals from the beginning of the $3 d$-series. Maybe the fact that the enhancement factors for core electrons in these metals are different in the lowand high-momentum regions $[2,12]$ makes finding of the proper function difficult.

Some systematics found in $G_{\mathrm{w}} F$ values in the results for all the metals we have examined hitherto is worth mentioning. The $G_{\mathrm{w}} F$, which amounts to over a dozen percent for the metals from $\mathrm{Fe}$ to $\mathrm{Cu}$, diminishes to about $7 \%$ in $\mathrm{Pd}$ and $\mathrm{Ag}[13,14]$ and drops to about $3 \%$ in Au [15]. The decreasing tendency in $G_{\mathrm{w}} F$ value when going from $3 d$ - through $4 d$ - to $5 d$-metals is not in compliance with the behaviour of respective $\lambda_{\mathrm{c}} / \lambda_{\exp }$ values, with $\lambda_{\mathrm{c}}$ from Ref. [12]. For V, Fe, Co, Ni and $\mathrm{Cu}$ the agreement, as we have shown above, is very good. The reason for the discrepancy for $4 d$ - and $5 d$-metals has not been found yet.

Let us draw some additional conclusions resulting from fitting ACAR data by the $P+F+G_{\mathrm{w}}$ model. Angular correlation data allow one to calculate the number $N_{\mathrm{NF}}$ of nearly free electrons per one metal atom according to the simple formula

$$
N_{\mathrm{NF}}=9.7 \times 10^{-4} A x_{\mathrm{p}}^{3} / \rho,
$$

where $A$ is the atomic mass, $\rho$ - substance density in $\mathrm{g} / \mathrm{cm}^{3}$, and $x_{\mathrm{p}}$ - parabola cut-off in mrad. The numbers $N_{\mathrm{NF}}$ calculated according to Eq. (1) are in Table II. They are in quite good agreement with the numbers of $s+p$ electrons in the solid state configuration of $3 d$-metals $[19,20]$ being close to 1.4 el/at. Discrepancy, as one could expect, appears for Sc, but a larger $N_{\mathrm{NF}}$ value for Ti should also be noticed. So, from the point of view of $N_{\mathrm{NF}}$, the model we have applied is very good.

To conclude we can state that our model is very good for $\mathrm{Fe}, \mathrm{Co}, \mathrm{Ni}$ and $\mathrm{Cu}$, i.e. for the metals from the second half of the series. The model allows one to find a contribution of the ionic-core electrons to the whole angular correlation curve. However a different model to describe the ACAR data for the metals from the beginning of the $3 d$-series should be searched.

The work was supported by the State Committee for Scientific Research (Republic of Poland) in the frame of the grant GBW/94/4/IFD.

\section{References}

[1] R.N. West, Solid State Commun. 9, 1417 (1971).

[2] M. Šob, in: Positron Annihilation, Eds. P.C. Jain, R.M. Singru, K.P. Gopinathan, World Scientific, Singapore 1985, p. 104.

[3] M. Šob, Solid State Commun. 53, 255 (1985).

[4] J.P. Carbotte, A. Salvadori, Phys. Rev. B 162, 290 (1967).

[5] S. Daniuk, G. Kontrym-Sznajd, J. Majsnerowski, M. Šob, H. Stachowiak, J. Phys. Condens. Matter 1, 6321 (1989).

[6] L. Oberli, A.A. Manuel, R. Sachot, P. Descouts, M. Peter, L.P.L.M. Rabou, P.E. Mijnarends, T. Hyodo, A.T. Stewart, Phys. Rev. B 31, 1147 (1985).

[7] M. Šob, in: Proc. 8th Annual Int. Symp. on Electronic Structure of Metals and Alloys, Gaussig, Germany, Ed. P. Ziesche, Tech. Universität Dresden, Dresden 1978, p. 170 . 
[8] M. Sob, in: Positron Annihilation, Eds. R.R. Hasiguti, K. Fujiwara, Japanese Institute of Metals, Sendai 1979, p. 309.

[9] P.E. Mijnarends, R.M. Singru, Phys. Rev. B 19, 6038 (1979).

[10] M. Sob, in: Proc. 10th Int. Conf. on Positron Annihilation, Beijing, China, May 23-29,1994, I 14 a, to be published.

[11] O. Johnson, Phys. Status Solidi B 99, 745 (1980).

[12] S. Daniuk, M. Šob, A. Rubaszek, Phys. Rev. B 43, 2580 (1991).

[13] A. Baranowski, E. Dębowska, Appl. Phys. A 51, 23 (1990).

[14] E. Dębowska, Mater. Sci. Forum 105-110, 635 (1992).

[15] E. Dębowska, Z. Phys. Chem. Neue Folge 181, 67 (1993).

[16] D.O. Welch, K.G. Lynn, Phys. Status Solidi A 77, 277 (1976).

[17] Xiong Liang-yue, private communication.

[18] A. Seeger, F. Banhart, W. Bauer, in: Positron Annihilation, Eds. L. Dorikens-Vanpraet, M. Dorikens, D. Segers, World Scientific, Singapore 1989, p. 275.

[19] O.K. Andersen, O. Jepsen, D. Glötzel, in: Highlights of Condesed Matter Theory, Eds. F. Bassini, F. Fumi, M. Tosi, North-Holland, Amsterdam 1985, p. 59.

[20] D.A. Papaconstantopoulos, Handbook of the Band Structure of Elemental Solids, Plenum, New York 1986. 